\title{
REVISIONES
}

\section{Zonas ribereñas: protección, restauración y contexto legal en Chile}

\author{
Riparian zones: Protection, restoration and legal context in Chile \\ Fabián I Romero ${ }^{\mathrm{a}, \mathrm{b}}$, Miguel A Cozano o,c, Rodrigo A Gangas ${ }^{\mathrm{a}, \mathrm{b}}$, Paulette I Naulin ${ }^{\mathrm{a}, \mathrm{b}^{*}}$

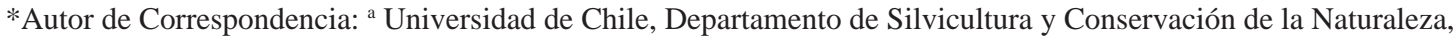 \\ Laboratorio de Biología de Plantas, Santa Rosa \# 11.315, Santiago, Chile, tel.: (02) 9785721- (02) 9785883, \\ fax (02) 5417971, Casilla 9206, pnaulin@uchile.cl \\ b Universidad de Chile, Facultad de Ciencias Forestales y Conservación de la Naturaleza, \\ Departamento de Silvicultura y Conservación de la Naturaleza, Santiago, Chile. \\ c Universidad de Chile, Facultad de Ciencias Agronómicas, Departamento de Ciencias Ambientales \\ y Recursos Naturales Renovables, Santiago, Chile.
}

\begin{abstract}
SUMMARY
Riparian zones are ecosystems dependent on courses or water bodies with a variable array of vegetation immersed in watersheds. These areas perform essential functions to the preservation of ecosystems and their spatial relations, influencing species richness and natural beauty, while providing goods and services for biodiversity and human welfare. Therefore, in any territorial unit, it is necessary to place the quality and relevance of riparian systems into context, given their importance as economic resources and providers of ecosystem services. However, the evaluation of the conservation status of these systems implies collecting those aspects that allow carrying out activities under current environmental regulations, guarantying the safekeeping of these systems in the long term. This paper explores the relevance of riparian zones in general and their participation in current Chilean legislation, with the aim of providing further information on their restoration and conservation. Chilean legislation identifies riparian zones as associated with ecosystem protection, but some weaknesses have been identified regarding the incorporation of the concept of restoration and the lack of financial incentives to carry out better protection. National Government should consider as a goal to create or adapt norms that allow protection as well as restoration of riparian systems, which would provide long-term viability to these ecosystems and their functions.
\end{abstract}

Key words: riparian vegetation, legislation, conservation, ecosystem services.

\section{RESUMEN}

Las zonas ribereñas, son ecosistemas dependientes de cursos o cuerpos de agua con una matriz variable de vegetación, inmersos en cuencas hidrográficas. Estas zonas cumplen funciones esenciales para la preservación de ecosistemas y sus relaciones territoriales, influyendo en el paisaje en términos de riqueza y belleza natural, a la vez que suministran bienes y servicios para la biota y el bienestar humano. Por lo tanto, en cualquier unidad territorial, es necesario determinar la calidad e importancia de los ecosistemas ribereños, ya que constituyen una fuente de recursos económicos y servicios ecosistémicos. No obstante, la evaluación del estado de conservación de estos sistemas implica recabar aquellos aspectos que permitan la ejecución de actividades bajo la normativa ambiental vigente, garantizando el resguardo de estos sistemas en una perspectiva a largo plazo. En este documento se analiza la relevancia de las zonas ribereñas en Chile, considerando la legislación actual, con el objetivo de aportar antecedentes en torno a su restauración y conservación. Se concluye que la legislación chilena identifica zonas ribereñas asociadas a la función de protección, existiendo algunas deficiencias en lo que respecta al concepto de restauración, y la falta de incentivos económicos para llevar a cabo una mayor protección, siendo necesario que el Estado cree o adecue normas que permitan la preservación de los ecosistemas ribereños y su restauración, incluyendo los recursos para incentivar estas acciones, lo cual otorgaría viabilidad de largo plazo a estos y a las funciones que cumplen.

Palabras clave: vegetación ribereña, legislación, conservación, servicios ecosistémicos.

\section{INTRODUCCIÓN}

Los ríos, lagos y cuerpos de agua en general, cumplen funciones esenciales para la preservación de los ecosistemas y las relaciones territoriales (Naiman et al. 1993, Allan 2004), teniendo una dependencia estrecha y recíproca con la vegetación ribereña (Guevara et al. 2008). Se reconoce esta última como sistema vegetal cercano a cursos y cuerpos de agua, siendo una transición entre hábitats terrestres y acuáticos en sus diferentes estratos verticales, incluidas las herbáceas. Weisberg et al. (2013) dan cuenta del papel ecológico significativo que cumple esta vegetación en la conservación de los servicios ecosistémicos asociados a las riberas. 
En general, estas zonas permiten al paisaje que los alberga procesar mayor cantidad de materia orgánica y capturar más nitrógeno, mejorar la cantidad y calidad del agua, facilitar el procesamiento de contaminantes y regular la temperatura y luz que ingresa a los sistemas acuáticos de mejor manera que aquellos sin este tipo de vegetación, reduciendo las probabilidades de afectar negativamente los ecosistemas río abajo (Scarsbrook et al. 2001, Sirombra y Mesa 2010). Además, los sistemas ribereños pueden categorizarse dentro de los más diversos, dinámicos y complejos hábitats presentes en la Tierra (Sweeney et al. 2004), frecuentemente fértiles y productivos, debido principalmente a su ubicación cercana a los cursos y cuerpos de agua, donde los depósitos aluviales proporcionan un suelo rico en nutrientes y materia orgánica (Granados et al. 2006).

Estos sistemas funcionan como complejas fuentes sustentadoras de biodiversidad, poseyendo límites, patrones sucesionales, disposiciones verticales en estratos, siendo además reservorios genéticos de las especies que los ocupan por el gran número de hábitats y microhábitats especiales, definidos por rasgos físicos (Granados et al. 2006), los que sirven tanto a especies acuáticas y semiacuáticas como a anfibios y especies derechamente terrestres (Naiman y Décamps 1997). Por ejemplo, diversos estudios indican, que existe una mayor densidad de aves en reproducción en los bosques ribereños que en las áreas cercanas de tierras altas (Granados et al. 2006).

Por otra parte, dada su naturaleza lineal, las zonas ribereñas o en galería, son efectivamente utilizadas como corredores biológicos, conectando bosques que en algunos casos se encuentran aislados, reduciendo el efecto de "isla" (Naiman et al. 1993). Algunos animales como la güiña (Leopardus guigna [Molina]), el colilarga (Sylviorthorhynchus desmursii [Gay]) y el churrín de la Mocha (Eugralla paradoxa [Kittlitz]), hacen uso de los corredores ribereños para movilizarse en terrenos fragmentados, disminuyendo los efectos adversos provocados por la fragmentación de hábitat (Carmona et al. 2011). Los paisajes enlazados con estos corredores, son capaces de soportar una variedad mucho mayor de fauna que una cuenca drenada por canales empobrecidos de vegetación, convirtiéndose en sistemas claves para el mantenimiento de la diversidad tanto faunística como florística (Granados et al. 2006, Guevara et al. 2008).

Con respecto a lo anterior, la dispersión de semillas por aves frugívoras y la depredación de semillas de especies leñosas por roedores, presenta diferencias significativas respecto a bosques de interior, siendo mayor y menor respectivamente en los bosques de ribera que en los bosques de interior (Carmona et al. 2011).

El número de individuos de macroinvertebrados fragmentadores de residuos orgánicos asociados a grandes coberturas de bosque nativo, es mayor en relación con bosques de bajas coberturas (Mancilla et al. 2009). La relevancia de esta diferencia se destaca al considerar que los fragmentadores son el eslabón primario en la degradación de los compuestos orgánicos mayores que ingresan a los cuerpos de agua, y por lo tanto, su desaparición podría provocar un "efecto cascada" en las demás etapas de la descomposición de residuos orgánicos.

Las alteraciones que provoca la introducción de especies exóticas sobre los sistemas ribereños, por ejemplo, el castor (Castor canadensis [Kuhl]), introducido al lado argentino de la Isla Grande de Tierra del Fuego en 1946, cuyos efectos negativos son derivados de la corta directa de árboles para forrajeo y la construcción de sus diques, conllevan cambios en los suelos y en la geomorfología ribereña (Arismendi et al. 2008).

El impacto del ganado en las zonas ribereñas incrementa la compactación del suelo, reduciendo la infiltración, degradando los márgenes de los cursos de agua y disminuyendo los niveles freáticos. Respecto a la vegetación, existe daño y reducción de la regeneración existente, y en algunos casos puede afectar especies endémicas asociadas a los cursos de agua. Sin embargo, no resulta fácil tratar estas alteraciones debido a que la actividad ganadera, generalmente, es el único medio de vida para los pobladores de estas áreas (Steinfeld et al. 2009).

Dada la presencia de estos servicios, algunas veces antagónicos, y retomando la estrecha relación entre la vegetación de ribera y los sistemas acuáticos, es necesario fortalecer el conocimiento acerca del estado de estos últimos, para velar por la salud de los ecosistemas de ribera (Allan 2004). En este sentido Lara et al. (2003, 2010), Little et al. (2009) y Huber et al. (2010) han mostrado que las microcuencas con porcentajes mayores de cobertura con plantaciones exóticas, producen un menor caudal estival que las cuencas con una cubierta mayor de especies nativas, afectando actividades productivas. $\mathrm{Al}$ respecto, las consecuencias de la introducción de estas especies (exóticas) pueden variar, desde provocar un ligero cambio en la composición de especies, hasta la extinción de plantas nativas, que generaría una profunda modificación del ecosistema (Parker y Reichard 1997, Sirombra y Mesa 2010). Sin embargo, estas actividades también generan impactos en los ecosistemas de ribera, pues como menciona Ochoa (2007), los conflictos ambientales ligados a la escasez de agua, como la extracción excesiva de agua con fines agrícolas, son los que mayormente generan cambios en el funcionamiento de los sistemas ribereños.

Lara et al. (2003) mencionan que en el caso de Chile, la provisión de agua en cantidad suficiente, libre de contaminación y de sedimentos, es esencial para asegurar la crianza de los salmones en sus primeros estados de desarrollo en las pisciculturas que usan agua de ríos. Por lo tanto, estas piscícolas dependen de los servicios ecosistémicos prestados por los bosques nativos adecuadamente manejados y conservados aguas arriba, de la misma manera que dependen la pesca o el turismo. Gualdoni et al. (2011) mencionan que la construcción de campings en las zonas ribereñas mejora la oportunidad del contacto humano con la naturaleza. 
Los ríos han constituido a lo largo de la historia focos preferenciales de concentración de poblaciones (Gualdoni et al. 2011). Como consecuencia, las comunidades humanas que se desarrollan en los márgenes de los cauces, ríos, arroyos y canales, someten constantemente a una gran presión a aquellos sistemas de los que dependen (Allan 2004, Guevara et al. 2008, Canizales et al. 2010), disminuyendo significativamente la diversidad tanto a nivel de abundancia como en la composición vertical en las zonas ribereñas, viéndose simplificados y atenuados los diferentes estratos, además de aumentar la mortalidad de plántulas en aquellos lugares perturbados (Canizales et al. 2010).

Dada esta relación estrecha y recíproca, entre la vegetación ribereña y la calidad de los cuerpos y cursos de agua, se vuelve relevante para el ser humano mantener la salud de los ecosistemas ribereños para preservar los servicios que estos proveen y la biodiversidad que sostienen (Allan 2004). Asimismo, el tipo de vegetación ribereña (nativa vs. exótica), influye sobre la cantidad y calidad del agua, y en el suministro de otros servicios ambientales, en sistemas acuáticos tanto costeros como montanos (Mancilla et al. 2009). En el centro-sur de Chile los bosques nativos han presentado un deterioro y disminución de los servicios ecositémicos, como consecuencia de prácticas forestales inadecuadas, incendios, sustitución por plantaciones de Pinus radiata D. Don o Eucalyptus spp. y habilitación agropecuaria (Lara et al. 2003, Echeverría et al. 2006, Little y Lara, 2010). Por ejemplo, el río Maullín es un sitio prioritario y de interés para la conservación in situ. No obstante, la tala y reemplazo por especies exóticas ha ido alterando su condición natural. Según Fernández et al. (2009), un 37 \% de las estaciones medidas tuvieron características de degradación extrema o alteración fuerte y calidad mala o pésima.

Por lo mismo, es importante no solo recabar información sobre el estado actual de estos sistemas, sino también sobre aquellos aspectos normativos y legales en torno a su conservación, en pos de garantizar la preservación de sus funciones ecosistémicas. En Chile, se han realizado algunas investigaciones que destacan la importancia de las zonas ribereñas mediante la aplicación de índices de calidad de ribera y de hábitat fluvial (Fernández et al. 2009, Palma et al. 2009). De igual manera se han realizado propuestas recientes con el ánimo de lograr una clasificación eco-hidrológica e hidro-morfológica de los sistemas dulceacuícolas chilenos (Peredo-Parada et al. 2011, Andreoli et al. 2012). Al respecto, en el presente trabajo se analizan y discuten los servicios que estos sistemas prestan a la sociedad y a la biodiversidad en general, además de estudiar su rol en la legislación vigente, analizando la efectividad de estos mecanismos en la conservación y restauración de estas zonas.

\section{RESTAURACIÓN ECOLÓGICA DE LOS ECOSISTEMAS RIBEREÑOS}

Como respuesta a la inminente pérdida de servicios ecosistémicos, la conservación y restauración de los eco- sistemas adquiere importancia dentro de los programas de investigación e inversión a nivel internacional, los cuales son desarrollados con el fin de mantener y recuperar la biodiversidad y los servicios prestados por estos ecosistemas (Young 2000).

La restauración ecológica se define como "el proceso de ayudar al restablecimiento de un ecosistema que ha sido dañado o destruido" (Society for Ecological Restoration- International Science and Policy Working Group, SER 2004). Martínez (1996) declara que la restauración ecológica se basa principalmente en la sucesión ecológica, siendo este su marco teórico fundamental. Lara et al. (2010) postulan que el concepto de restauración se debería relacionar con "bonificar" las acciones tendientes a recuperar atributos de estructura y funcionamiento de los ecosistemas que se han deteriorado y perdido.

Los ecosistemas restaurados pueden mejorar la provisión de servicios ecosistémicos y ayudar en la conservación de la biodiversidad, siendo necesario observar que dichas acciones de restauración se ajusten a la composición y estructura original de la cubierta vegetal, incluyendo las dinámicas de estos sistemas (Holl y Aide 2011). Al respecto, Meli (2003) menciona distintos factores que limitan la regeneración natural de bosques sobre terrenos degradados, como la composición y densidad del banco y la lluvia de semillas, la capacidad germinativa de las distintas especies, la supervivencia y crecimiento de plántulas, las condiciones microclimáticas y edáficas del establecimiento, y las interacciones de las plántulas del bosque con la vegetación remanente.

La restauración de los ecosistemas depende fuertemente del nivel de degradación de la vegetación y el suelo, de la vegetación remanente y de los objetivos de la intervención, los cuales deben incorporar los procesos físicos y biológicos que influyen en estos ecosistemas, y los tipos de perturbaciones que los afectan (Goodwin et al. 1997), además de los modos culturales y las necesidades de las comunidades locales (Holl y Aide 2011).

Sobre el restablecimiento de las funciones intrínsecas de los ecosistemas, Naiman y Décamps (1997) mencionan que la restauración de ecosistemas ribereños permite la diversificación de hábitats para la vida silvestre tanto terrestre como acuática, la generación de corredores para animales que dispersan semillas y un incremento de la materia orgánica sobre el sustrato. A su vez, mejora la calidad visual y las oportunidades de recreación a través del paisaje.

Desde el punto de vista de servicios prestados por estos sistemas, se puede mencionar la provisión de agua, normalmente vista solo como un servicio ecosistémico a conservar o mantener en las cuencas, pero no a recuperar (Little y Lara 2010), por lo que la aplicación de medidas para el resguardo y restauración de la provisión de agua como servicio ecosistémico generaría gran impacto (Little et al. 2009). En este aspecto, se espera que en Chile se desarrollen programas destinados a la investigación para la elaboración de mejores pautas de manejo de sistemas 
boscosos a nivel de cuenca y la protección de las zonas ribereñas (Little et al. 2009).

En muchos casos se presenta la necesidad de establecer franjas de protección cercanas a los cursos de agua, pero la pregunta que subyace es qué tamaño deben tener estas. En este sentido, Gayoso y Gayoso (2003) señalan que muchas veces la razón de la franja de protección se sustenta en la conservación de la biodiversidad y en la habilitación de corredores biológicos para la vida silvestre. Sin embargo, es difícil establecer esta franja a priori, pues no existe consenso sobre su tamaño ideal, así como tampoco si debiese ser un ancho variable o fijo, puesto que estas dimensiones dependerán en gran medida de las necesidades y objetivos de conservación o manejo aplicados a la zona en cuestión, y de la persistencia de los usos del suelo a lo largo de las microcuencas y de los sistemas acuáticos más complejos (e.g. ríos, humedales).

\section{CONTEXTO LEGAL EN CHILE}

La legislación vinculada con la vegetación ribereña es la forestal, la cual se caracteriza por ser abundante, estar dispersa en diferentes cuerpos legales y reglamentarios que responden a épocas pasadas, con diversas contradicciones, incoherencias, puntos incompletos y, a veces, "vencimientos" en cuanto a los actuales objetivos (Gallardo 2000). En Chile, desde muy temprano se regula sobre la protección de los cauces y las zonas ribereñas (cuadro 1). Por ejemplo, el Decreto No 4.363 del año 1931, conocida como Ley de Bosques, en su artículo 5, establece la prohibición de "(1) la corta de árboles y arbustos nativos situados a menos de $400 \mathrm{~m}$ sobre los manantiales que nazcan en los cerros y los situados a menos de $200 \mathrm{~m}$ de sus orillas desde el punto en que la vertiente tenga su origen hasta aquel en que llegue al plano y (2) la corta o destrucción del arbolado situado a menos de $200 \mathrm{~m}$ de radio de los manantiales que nazcan en terrenos planos no regados..." (Gayoso y Gayoso 2003, Pellet et al. 2005).
Diversos reglamentos de la Ley de Bosques permanecen vigentes, aunque con un alto grado de ineficacia e incumplimiento, volviéndose necesaria su derogación, aludiendo a que el artículo $5 \mathrm{y}$ los reglamentos orientados a especies determinadas se han vuelto ineficientes ante los tipos forestales definidos en el Reglamento Técnico del Decreto Ley (DL) 701 (Gallardo 2000). En cambio otros autores, como Pellet et al. (2005), reconocen por ejemplo, su directa relación con el manejo de cuencas, ya que posibilita la protección del suelo y el agua, utilizando prohibiciones de corta sobre la vegetación.

Según Gayoso y Gayoso (2003), "la no consideración de las diferencias topográficas, relieves, suelos, variables climáticas, vegetación, tipo e importancia de los cauces han hecho inaplicable la norma". También indican que "para suplir este déficit, la institucionalidad forestal ha ido incorporando en las normas de manejo, orientaciones respecto del tratamiento de la protección de cauces”, mencionando las siguientes normas de manejo:

- Normas de manejo aplicables a faenas de raleo para el tipo forestal roble-raulí-coigüe. Se establece que los cursos de agua permanentes contarán con una franja de protección de $30 \mathrm{~m}$ de ancho a cada lado del afluente. Esta franja no podrá ser intervenida. Incluso no se permite la caída de árboles desde áreas vecinas que estén siendo intervenidas. Respecto de los cursos de agua no permanentes, se protegen con una franja de $15 \mathrm{~m}$ de ancho como mínimo en sectores que están siendo intervenidos. También se protege de cualquier intervención, las áreas de pendientes iguales o superiores a 60 $\%$ por más de $30 \mathrm{~m}$ (Gayoso y Gayoso 2003, Pellet et al. 2005).

- Normas de manejo aplicables a la corta final en el tipo forestal siempreverde. Fija en 10 a $20 \mathrm{~m}$ el ancho mínimo de las franjas de protección en cursos de agua no permanentes y entre 20 - $30 \mathrm{~m}$ para los permanentes, según la variación de pendiente lateral entre menos de

Cuadro 1. Legislación relacionada a la protección del cauce y la vegetación asociada a cursos de agua en Chile. Legislation related to the protection of water bodies and courses, and vegetation associated with streams in Chile.

\begin{tabular}{ll}
\hline \multicolumn{1}{c}{ Cuerpo legal } & \multicolumn{1}{c}{ Característica principal de interés } \\
\hline $\begin{array}{l}\text { Ley 11.402 de 1953 Sobre las obras de defensa y } \\
\text { regularización de las riberas y cauces de los ríos, lagunas y } \\
\text { esteros. }\end{array}$ & $\begin{array}{l}\text { Regula la extracción de ripio y arena en los cauces de los ríos y } \\
\text { esteros (artículo 11). }\end{array}$ \\
\hline $\begin{array}{l}\text { Ley 18.378 de 1984 Sobre distritos de conservación de } \\
\text { suelos, bosques y aguas; y conservación de la riqueza } \\
\text { turística. }\end{array}$ & $\begin{array}{l}\text { Establece que el Presidente de la República puede decretar la } \\
\text { prohibición de cortar los árboles situados hasta a cien metros (100 } \\
\text { m) de las orillas de ríos y lagos que sean bienes nacionales de uso } \\
\text { público, como también, en quebradas (artículo 4). }\end{array}$ \\
\hline $\begin{array}{l}\text { Ley 18.892 de 1991 Ley general de pesca y acuicultura. } \\
\text { Ley 19.561 de 1998 Modifica el DL No 701, de 1974, sobre } \\
\text { Fomento Forestal. }\end{array}$ & $\begin{array}{l}\text { Indica las disposiciones para la preservación y conservación de los } \\
\text { recursos hidrobiológicos. }\end{array}$ \\
\hline
\end{tabular}


Ley 20.017 del 2005 Modifica el código de aguas.

Ley 20.283 del 2008 Sobre recuperación del bosque nativo y fomento forestal.

Ley 20.417 del 2010 Crea el Ministerio, el Servicio de Evaluación ambiental y la Superintendencia del Medio ambiente.

DFL 1600 de 1931 y Decreto 1600 de 1931 Sobre constitución de la propiedad Austral.

DFL 1.122 de 1981 Fija texto del código de aguas.

DL Nº 3.485 de 1981 Aprueba convención relativa a las zonas de importancia internacional, especialmente como hábitat de las aves acuáticas, firmada en Ramsar, Irán, el 2 de febrero de 1971.

DS Nº 4.363 de 1931 Ley de bosques.

DS No 2.3741937 Aprueba el reglamento para la explotación de bosques existentes en las cuencas hidrográficas.

DS No 259 de 1980 Reglamento técnico del DL 701 de 1974.

DS No 771 de 1981 Promulga la convención sobre zonas húmedas de importancia internacional especialmente como hábitat de las aves acuáticas, suscrito en Irán el 2 de febrero de 1971.

DS N ${ }^{\circ} 971$ de 1987 Protocolo para enmendar la convención sobre zonas húmedas de importancia internacional especialmente como hábitat de las aves acuáticas.

DS Nº 30 de 1997 Reglamento del Sistema de Evaluación de Impacto Ambiental.

DS No 609 de 1978 Fija normas para establecer deslindes propietarios riberanos con el bien nacional de uso público por las riberas de los ríos, lagos y esteros.

DS Nº 193 de 1998 Reglamento general DL 701.

DS No 1.341 de 1998 Reglamento que establece normas contables aplicables a los contribuyentes que realizan actividades forestales de conformidad al DL 701 de 1974.

DS No 192 de 1998 Reglamento para el pago de las bonificaciones forestales.

Decreto $\mathrm{N}^{\circ} 82$ de 2011 Reglamento de suelos, aguas y humedales de la Ley $\mathrm{N}^{\circ} 20.283$.

Decreto N 14 del 2013 Aprueba reglamento para la determinación del “Caudal ecológico mínimo”.
Establece cambios para fijar el texto del código de aguas. (DFL 1.122).

Define los "bosques nativos de conservación y protección" para el resguardo de suelos y recursos hídricos (artículo 2, 5).

Indican las funciones del Ministerio, entre ellas, proponer políticas ambientales y aprovechamiento de recursos hídricos (artículo 70).

Establece que el propietario cede gratuitamente al Fisco una faja hasta de 25 metros de terreno firme en la ribera de los ríos y lagos (artículo 34).

Fija disposiciones relacionadas al dominio y aprovechamiento de las aguas (Título II).

Promueve la conservación de las zonas húmedas como reguladoras de los regímenes de agua y como regiones que permiten la conservación de una flora y fauna características, especialmente aves acuáticas.

Prohíbe la corta o destrucción del arbolado próximo a cursos de agua (artículo 5).

Prohíbe la destrucción del equilibrio hidrológico-forestal, a través de un sistema de explotación de bosques insertos en una cuenca y establecidos sobre terrenos declarados forestales (artículo 4) (Pellet et al. 2005).

Establece los métodos de corta y sus disposiciones en bosque nativo.

Promueve la protección de humedales en Chile.

Promueve la protección de humedales en Chile.

Hace referencia al permiso de ejecutar labores mineras en lugares donde se incluyen cursos de agua y lagos de uso público. Indica la restauración o reparación del área intervenida, en los casos que corresponda (letra d, artículo 85).

Fija normas para establecer deslindes de los cauces de los ríos, lagos y esteros, de oficio cuando las circunstancias así lo exigieren o a petición del propietario riberano cuando éste lo solicite (número 3).

Establece los puntos a incluir en planes de manejo, declara bosques de protección y estudios técnicos para calificar terrenos APF.

Menciona la exención de Impuesto territorial para terrenos cubiertos con bosque nativo o bosques de protección (artículo 5, en conformidad con artículo 13 del Decreto Ley 701).

Indica las actividades a bonificar en conformidad con el DL 701.

Define Zonas de protección y actividades que prohíben la eliminación o menoscabo de la vegetación asociada a cauces y cuerpos de agua.

Determina la cantidad mínima de agua que debe tener una fuente superficial, como un río o un lago, para mantener en buenas condiciones la diversidad biológica del cauce, sin afectar su desarrollo ni el patrimonio ambiental del país.

Fuente: elaboración propia basada en Pellet et al. (2005) y documentos de la Biblioteca del Congreso Nacional de Chile, 2013. 
$30 \%$ y más de $45 \%$ (Gayoso y Gayoso 2003, Pellet et al. 2005).

- Normas aplicables al manejo de plantaciones de Eucalyptus spp., Pinus radiata D. Don y otras especies exóticas. Señalan que cuando el área a intervenir colinde con cursos de aguas permanentes y temporales, se conservará una franja de bosque de protección cuyo ancho será de $25 \mathrm{~m}$ a cada lado del curso de agua (Gayoso y Gayoso 2003).

Otro ejemplo es el Decreto Supremo No 2.374, que aprueba el reglamento para la explotación de bosques existentes en las cuencas hidrográficas. En su artículo 4 señala que: "En todas aquellas situaciones en que el terreno sea de excesiva pendiente, o en que la naturaleza de él sea muy desagregable, se impedirá en absoluto la explotación forestal, por lo menos en una zona no menor de $200 \mathrm{~m}$ a un lado y otro del "talweg (línea que une los puntos más bajos de una quebrada)". Este reglamento se orienta a evitar la destrucción del equilibrio hidrológico-forestal, a través de un sistema de explotación de bosques insertos en una cuenca y establecidos sobre terrenos declarados forestales (Pellet et al. 2005).

Uno de los instrumentos más utilizados en los últimos años, corresponde al Decreto Ley 701 (1974), sobre fomento forestal, modificado por la Ley 19.561 de 1998. Este Decreto, en su artículo 13 identifica como bosques de protección "aquellos ubicados en suelos frágiles con pendientes iguales o superiores a $45 \%$ y los próximos a fuentes, cursos o masas de agua destinados al resguardo de tales recursos hídricos, los cuales podrán cubrir una franja equivalente al ancho máximo del cauce natural, la que no podrá exceder de 400 metros medidos desde el borde del mismo". Sin embargo, no define medidas de conservación para estos bosques. Por otra parte, la Ley 20.283 de 2008, sobre Recuperación del Bosque Nativo y Fomento Forestal, define en el artículo 2 que un "bosque nativo de conservación y protección es aquel, cualquiera sea su superficie, ubicado en pendientes iguales o superiores a $45 \%$, en suelos frágiles o a menos de 200 metros de manantiales, cuerpos o cursos de agua naturales, destinados al resguardo de tales suelos y recursos hídricos".

Las definiciones de bosque de protección, emitidas por el DL 701 y la Ley 20.283, muestran similitudes en cuanto a las especificaciones de suelo y pendiente, sin embargo, existen diferencias notorias. Por ejemplo, un bosque nativo que se extiende $300 \mathrm{~m}$ desde el borde de un río sólo es considerado "bosque nativo de conservación y protección" hasta antes de sobrepasar los 200 m medidos desde el borde del cauce según la Ley 20.283. Sin embargo, para el mismo caso, el DL 701 establece la totalidad de extensión como bosque de protección. Dada esta diferencia, Granados et al. (2006) indican que resulta preferible "definir ecológicamente el borde o límite exterior de la zona de amortiguamiento" en la mayoría de los tipos de manejo. Esto se relaciona al "ancho máximo del cauce natural" que hace referencia el DL 701, especificándolo como un parámetro de medición, algo que se omite en la definición de bosque nativo de conservación y protección.

El reglamento de Suelos, Aguas y Humedales de la Ley 20.283, publicado en febrero de 2011, establece en el artículo 2 letra p) una "zona de protección de exclusión de intervención”, cuya amplitud dependerá del área de sección de cauce (figura 1A). El mismo reglamento, también establece en el artículo 2 letra q) una "zona de protección de manejo limitado" contigua a la zona de exclusión de intervención de un curso natural de agua de sección mayor a $0,50 \mathrm{~m}^{2}$, cuerpo de agua o manantial, cuya amplitud variará con las pendientes presentes en el terreno (figura 1B). Además, en el artículos 3 y 4, se indican las intervenciones posibles de realizar y las restricciones a considerar en cada zona de protección, mientras que el artículo 5 regula la aplicación de estas actividades en territorio nacional dependiendo de la región. La figura 1C muestra cómo se verían ambas zonas de protección rodeando un curso de agua. Por otra parte, el mismo reglamento establece en torno a los cuerpos de agua presentes en Sitios Prioritarios de Conservación o sitios Ramsar, una zona de protección de $10 \mathrm{~m}$ de ancho medidos horizontalmente, a partir del cual se podrá intervenir la cobertura arbórea, dejando al menos un $50 \%$ de esta.

Respecto a las bonificaciones, la Ley 20.283 incluye y promueve en el artículo 22: 1) las actividades que favorezcan la regeneración, recuperación o protección de formaciones xerofíticas de alto valor ecológico o bosques nativos de preservación, con el fin de lograr la mantención de la diversidad biológica, con excepción de aquellos pertenecientes al Sistema Nacional de Áreas Silvestres Protegidas por el Estado; 2) las actividades silviculturales dirigidas a la obtención de productos no madereros; 3 ) las actividades silviculturales destinadas a manejar y recuperar bosques nativos para fines de producción maderera. $\mathrm{Al}$ respecto, Lara et al. (2010) comentan que un defecto conceptual de la Ley 20.283 es que no incorpora ni privilegia las bonificaciones a las plantaciones en las zonas de protección ribereña.

En el país existen experiencias relevantes de iniciativas de conservación, estando entre las más notables el establecimiento y desarrollo de las áreas protegidas privadas y la certificación de las plantaciones. Este proceso ha ocurrido principalmente por iniciativa de las empresas forestales ante la demanda de los consumidores por productos certificados, destacando los dos grandes "conglomerados de empresas forestales de Chile (Forestal Arauco S.A. y CMPC) que requieren hacer modificaciones a fin de lograr su certificación por el FSC (Forest Stewardship Council)" (Lara et al. 2010).

\section{DISCUSIÓN}

La protección de la vegetación ribereña presenta beneficios directos para los seres humanos. El suministro de 


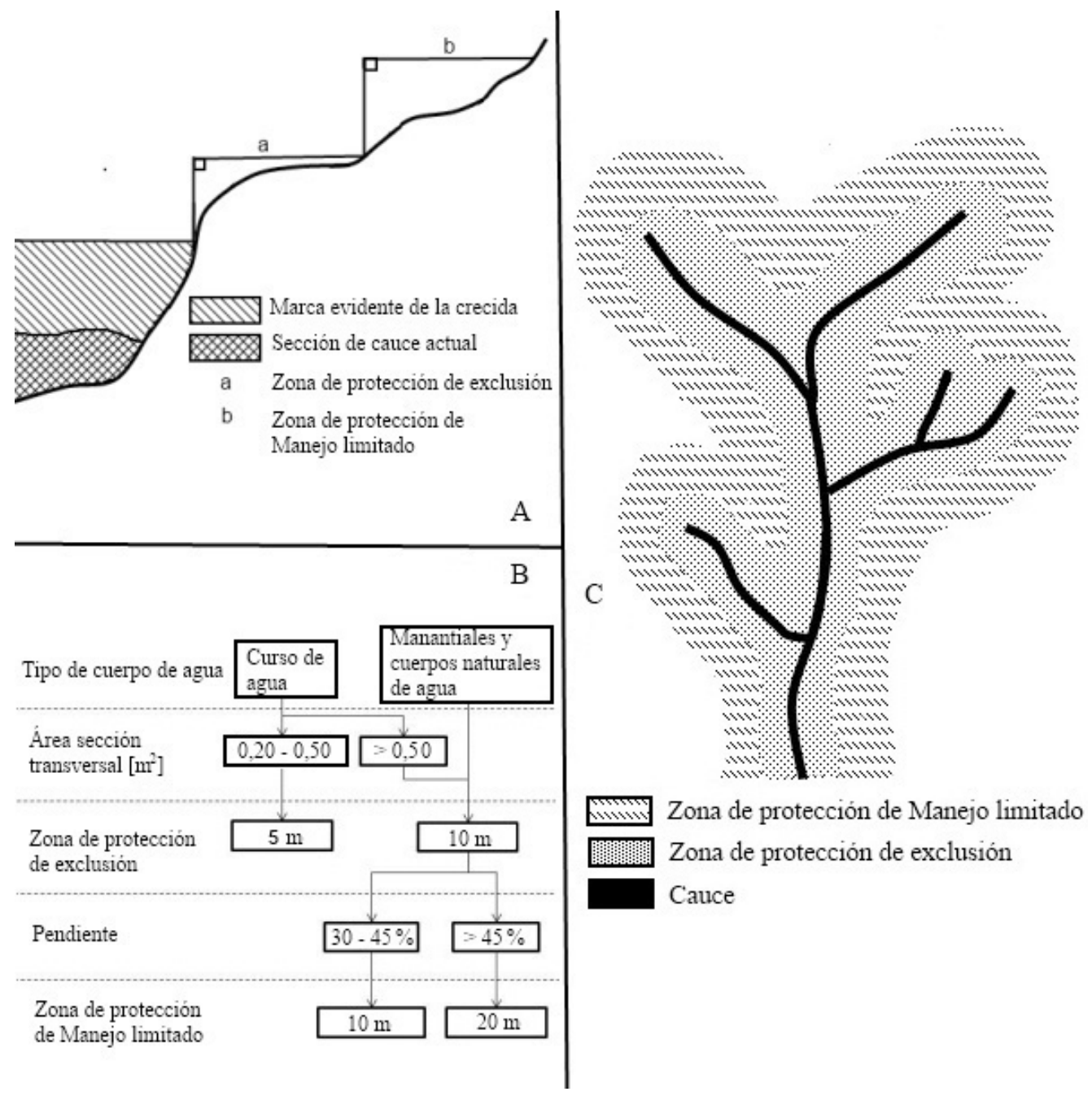

Figura 1. Descripción y caracterización de la normativa vigente en Chile en relación con la protección de los cuerpos de agua, según el reglamento de Suelo, Agua y Humedales de la Ley 20.283. A) Ejemplo de sección transversal de un curso de agua. B) Esquema del actual Reglamento de Suelo, Agua y Humedales (Ley 20.283) que señala el establecimiento de zonas de protección para distintos cuerpos de agua. C) Diagrama de la disposición final de las zonas de protección.

Description and characterization of the law in force in Chile related to the protection of water bodies based on the Norm of Soil, Water and Wetlands of Law 20,283. A) Example of Cross Section of a stream. B) Outline of the current regulation including establishment of protection zones for different water bodies. C) Diagram of the final disposition of the Protection Zones.

agua como servicio ecosistémico depende de la cuenca hidrográfica, lugar donde se conjugan los elementos que conforman un ecosistema y se desarrollan los procesos que la regulan. Por lo tanto, la estructura, funcionamiento y estado de conservación de la cuenca determinarán en qué medida se concreta la provisión de este servicio (Little et al. 2009). Las zonas ribereñas, son buena fuente de provisión de agua de mejor calidad, de valor paisajístico y turístico, además de cumplir funciones ecológicas como corredores biológicos y sustentadores de la biodiversidad, lo que las hace elementos claves para apoyar la decisión de protegerlas aún más, diseñando estrategias de manejo destinadas a un uso más eficiente y racional de estos servicios (Fernández et al. 2009).
Los incentivos a la restauración y protección del bosque en las zonas ribereñas incluyen bonificaciones, exención de impuestos o pago por servicios ambientales (Kosoy et al. 2007) constituyendo un impulso tanto para las grandes empresas que buscan obtener la certificación y una contribución a una demanda de la sociedad en la actualidad, como para pequeños propietarios de bosques nativos en las zonas rurales. Sin embargo, es fundamental la realización de estudios científicos que caractericen la vegetación, la dinámica de la regeneración y la propagación de plantas en las zonas ribereñas para la correcta implementación de actividades de recuperación y restauración de estos ecosistemas de características singulares en cuanto a la distribución espacio-temporal de sus componentes biológicos. 
Además, es necesario estudiar si los anchos establecidos en el reglamento de Suelo, Aguas y Humedales de la Ley de Bosque Nativo 20.283 son adecuados, identificando los efectos positivos y negativos que ocasionaría el aumentar el ancho de las zonas de protección establecidas en dicho reglamento de acuerdo a lo recomendado en apartados anteriores, así como también se debe indagar sobre la restauración en las cabeceras de cuencas, ya que estas regulan naturalmente los recursos hídricos, además de controlar los flujos de sedimentos río abajo (Forget et al. 2013). Las cabeceras de cuencas solo están mencionadas en el reglamento de Suelo, Aguas y Humedales de la Ley de Bosque Nativo 20.283, en lo referido a la protección en la cabecera y borde de las cárcavas, prohibiendo la corta y destrucción de árboles y arbustos en bosque nativo a menos de $5 \mathrm{~m}$.

El fortalecer la legislación actual para apoyar el desarrollo de mecanismos de conservación y restauración realmente efectivos, esperando que se desarrollen planes, políticas y programas para la protección y restauración ecológica en las zonas ribereñas, manteniendo una extensión apropiada de las cubiertas vegetales en la cabecera de las cuencas, sería uno de los aspectos fundamentales para asegurar el equilibrio dinámico de la cuenca y su aprovechamiento sostenido a largo plazo (Mintegui y Robredo 1994). Estas actividades se complementan con otras propuestas recientes sobre la caracterización eco-hidrológica e hidro-morfológica de los ríos chilenos (Peredo-Parada et al. 2011 y Andreoli et al. 2012).

Respecto de falencias presentes en la legislación, el artículo 5 del Decreto 4.363 del año 1931 indica que "no se podrá cortar en dichos sectores...”, no obstante en conformidad a lo establecido por el Decreto Ley 701 de 1974 "se puede realizar la corta en estos sectores si hay de por medio una justificación que lo valide". Sin embargo, cabe preguntarse ¿Cuáles son aquellas causas justificadas? Sería entonces necesario detallar aquellas "causas" (Gayoso y Gayoso 2003, Pellet et al. 2005).

La creación de una política pública que se encargue de velar específicamente por la integridad de los ecosistemas de ribera, dados los múltiples bienes y servicios que otorga tanto para el bienestar de los sistemas naturales como para el ser humano es fundamental. Se recomienda revisar en detalle la propuesta de incluir explícitamente bonificación a acciones de protección y restauración de la vegetación de ribera, considerándose la vegetación en todos los estratos verticales circundante a cuerpos y cursos de agua permanentes o estacionales, destacando así la importancia de generar proyectos destinados a recuperar no sólo la estructura sino las funciones y los servicios ecosistémicos de la vegetación nativa asociada a los sistemas ribereños. Es decir, una oportunidad para aumentar la protección de la vegetación asociada a cursos de agua surge al incluir, en las actividades susceptibles de bonificación, la restauración con especies nativas en franjas ribereñas, cuando estas se encuentren desprovistas de vegetación arbórea o degradadas.

Todos los reglamentos referentes a la reforestación (cuadro 1) que apuntan a la restauración no especifican el tipo de flora y vegetación a utilizar en dicha reforestación, pudiendo utilizarse especies exóticas como Pinus radiata y Eucalyptus spp., cuya demanda hídrica es mayor en comparación con especies del bosque nativo, recomendándose poner especial atención a las especies con que se restaurarán las riberas, la revegetación con especies exóticas, podría traer más desventajas que ventajas (Huber et al. 2010).

Huber et al. (2010) establecen que para el caso de comunidades del sector rural que viven en microcuencas abastecedoras de agua, resulta fundamental la asistencia técnica y capacitación dirigida a mejorar sus prácticas productivas, siendo necesario prohibir cualquier actividad productiva en estas zonas que pueda contaminar los cuerpos y cursos de agua aledaños, invirtiendo además en la infraestructura necesaria para evitar las alteraciones del canal y la fragmentación del bosque, así como la interrupción de las interacciones ecológicas, permitiendo la continuidad longitudinal entre el ecosistema acuático y su entorno (Gualdoni et al. 2011). Esto con el fin de asegurar la protección y restauración de la vegetación aledaña a los cursos de agua, un mayor ancho en las zonas de protección ribereña resultará en una menor cantidad de sedimentos transportados, aumentando y mejorando la provisión de agua de calidad para el consumo humano.

El pago por servicios ambientales se perfila como una posibilidad a la hora de conservar los servicios prestados por estos ecosistemas. De acuerdo con estos últimos, es importante señalar que bajo lo reglamentado por el DL No 701 y por la Ley 20.283, se considerarán como pequeños predios forestales aquellos cuya superficie no exceda, como regla general, las 200 hectáreas, donde sus activos no superen las 3.500 UF y estas sean trabajadas de forma directa por el propietario (Gayoso et al. 2010).

El artículo 22 de la Ley 20.283, respecto al "Fondo de Conservación, Recuperación y Manejo del Bosque Nativo" señala que se financiarán, entre otras actividades, aquellas silviculturales dirigidas a la obtención de productos forestales no madereros. Es posible acoger pago por servicios ambientales dentro de la normativa de los fondos concursables de la Ley 20.283, pudiendo agregarlos dentro de la categoría de bonificación para la obtención de productos forestales no madereros, entendiendo los pagos por servicios ambientales como intangibles que otorgan un beneficio al ser humano, constituyéndose en una nueva forma de protección ambiental, y, en este caso, una forma de proteger los ecosistemas ribereños (Gayoso et al. 2010).

Las acciones correctivas para la conservación y restauración de la vegetación existente en áreas cercanas a las riberas de los cursos y cuerpos de agua, que la ley incluye entre los "bosques de protección" se implementarían con mayor celeridad por parte de las empresas, si estas fueran bonificadas, representando además una ganancia para su certificación ambiental (Lara et al. 2010), lo que permitiría un mejoramiento continuo en las prácticas de manejo forestal, además de la entrega de información sobre la gestión de los bosques a clientes y otras partes interesadas, 
demostrando que el bosque que es manejado de manera sustentable, se traduce en un aumento de la cadena de valor para la empresa y en un bien público que beneficia a la sociedad en su conjunto.

\section{CONCLUSIONES}

Las zonas ribereñas son sistemas de alta complejidad. Los bosques asociados a cursos de agua cumplen roles de estabilización, fuente de materia y energía, refugio de fauna, filtro de nutrientes, regulador de la temperatura y productividad del agua, conexión entre paisajes y protección contra la erosión, entre otros. Dada las características de estos sistemas, es necesario comprender a cabalidad su desarrollo, el medio en que estos se encuentran insertos y su relación con el ambiente circundante.

La legislación en Chile identifica zonas ribereñas asociadas con la función de protección, pero tiene debilidades en cuanto a la ausencia del concepto de "restauración". La Ley 20.283 no considera explícitamente acciones a bonificar en las zonas de protección. El Estado debe cumplir un rol primario en la protección y recuperación de la vegetación nativa aledaña a los cuerpos y cursos de agua, a escala de cuenca hidrográfica y según los diferentes usos del suelo, dada las funciones y servicios ecosistémicos que ellos brindan a la sociedad. También es necesario crear o adecuar normas específicas para fomentar la restauración en las cabeceras y bordes de los sistemas acuáticos, principalmente en arroyos y ríos, restauración que debe estar dirigida de acuerdo con los patrones naturales de perturbación, sucesión ecológica, composición y estructura de la vegetación característica de cada localidad y acorde con los diferentes usos del suelo.

\section{AGRADECIMIENTOS}

Los Autores agradecen a la académica de la Universidad de Chile, Facultad de Ciencias Forestales y de la Conservación de la Naturaleza; Dra. Rosa Scherson, y Dr. Sergio Estay, académico de la Universidad Austral de Chile, Instituto de Ciencias Ambientales y Evolutivas, así como a Dr.(c) Abraham Albornoz, por sus revisiones, aclaraciones y consejos para mejorar este documento. Se agradece el apoyo del Laboratorio de Biología de Plantas del Departamento de Silvicultura y Conservación de la Naturaleza, Universidad de Chile.

\section{REFERENCIAS}

Allan J. 2004. Landscapes and Riverscapes: The influence of Land use on Stream Ecosystems. Annual Review of Ecology, Evolution, and Systematics 35:257-284.

Andreoli A, L Mao, A Iroumé, JL Arumí, A Nardini, R Pizarro, D Caamaño, C Meier, O Link. 2012. The need for a hydromorphological approach to Chilean river management. Revista Chilena de Historia Natural 85(3): 339-343.
Arismendi I, P Szejner, A Lara, M González. 2008. Impacto del castor en la estructura de bosques ribereños de Nothofagus pumilio en Tierra del Fuego, Chile. Bosque 29(2): 146-154.

Canizales P, G Alanís, S Favela, M Torres, E Alanís, J Jiménez, H Padilla. 2010. Efecto de la actividad turística en la diversidad y estructura del bosque de galería en el noreste de México. Ciencia UANL 13(1): 55-63.

Carmona M, J Aravena, M Bustamante-Sánchez, J Celis-Diez, A Charrier, I Díaz, J Díaz-Forestier, M Díaz, A Gaxiola, A Gutiérrez, C Hernandez-Pellicer, S Ippi, R Jaña-Prado, P Jara-Arancio, J Jimenez, D Manuschevichi, P Necochea, M Nuñez-Avila, C Papic, C Pérez, F Pérez, S Reidi, L Rojas, B Salgado, C Smith-Ramírez, A Troncoso, R Vásquez, M Willson, R Rozzi, J Armesto. 2011. Estación Biológica Senda Darwin: Investigación ecológica de largo plazo en la interfase ciencia-sociedad. Revista Chilena de Historia Natural 83(1): 113-142.

Echeverría C, D Coomes, J Salas, J Rey Benayas, A Lara, A Newton. 2006. Rapid deforestation and fragmentation of Chilean temperate forests. Biological Conservation 130: 481-494.

Fernández L, J Rau, A Arriagada. 2009. Calidad de la vegetación

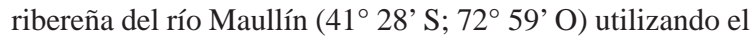
índice QBR. Gayana Botánica 66(2): 269-278.

Forget G, C Carreau, D Le Coeur, I Bernez. 2013. Ecological Restoration of headwaters in a rural landscape (Normandy, France): a passive approach taking hedge networks into account for riparian tree recruitment. Restoration Ecology 21(1): 96-104.

Gallardo E. 2000. Descripción general de la legislación forestal vigente en Chile. Legislación Forestal y Áreas Silvestres Protegidas, Editorial ConoSur. In Valores ambientales del territorio. Consultado 25 jul. 2013. Disponible en http://www. achidam.cl/Achidam_reportes_valoresambientales.html

Gayoso J, S Gayoso. 2003. Diseño de zonas ribereñas: Requerimientos de un ancho mínimo. Valdivia, Chile. Universidad Austral de Chile. Facultad de Ciencias Forestales. 12 p.

Gayoso S, Cordero D, Obreque F, Vergara G. 2010. El Estado del arte del pago por Servicios Ambientales en Chile. Valdivia, Chile. Instituto Forestal (INFOR). 29 p.

Goodwin C, C Hawkins, J Kershner. 1997. Riparian restoration in the western United States: Overview and perspective. Restoration Ecology 5(4): 4-14.

Granados D, M Hernández, G López. 2006. Ecología de las zonas ribereñas. Revista Chapingo. Serie Ciencias Forestales y del Ambiente 12(1): 55-69.

Gualdoni C, C Duarte, E Medeo. 2011. Estado ecológico de dos arroyos serranos al sur de Córdoba, Argentina. Ecología Austral 21(2): 149-162.

Guevara G, G Reinoso, JE García, L M Franco, L J García, D C Yara, N Briñez, M Ocampo, MI Quintana, DY Pava, NY Flórez, MF Ávila, EE Hernández, LA Lozano, M Guapucal, DA Borrero, EJ Olaya. 2008. Aportes para el análisis de ecosistemas fluviales: una visión desde ambientes ribereños. Revista Tumbaga 3: 109-127.

Holl K, T Aide. 2011. When and where to actively restore ecosystems? Forest Ecology and Management 261(10): 15581563.

Huber A, A Iroumé, C Mohr, C Frêne. 2010. Efecto de las plantaciones de Pinus radiata y Eucalyptus globulus sobre el recurso agua en la Cordillera de la Costa de la Región del 
Biobío, Chile. Bosque 31(3): 219-230.

Kosoy N, M Martínez-Tuna, R Muradian, J Martínez-Alier. 2007. Payments for environmental services in watersheds: Insights from a comparative study of three cases in Central America. Ecological Economics (61): 446-455.

Lara A, D Soto, J Armesto, P Donoso, C Wernli, L Nahuelhual, F Squeo. 2003. Componentes Científicos Clave para una Política Nacional Sobre Usos, Servicios y Conservación de los Bosques Nativos Chilenos. Valdivia, Chile. Iniciativa Científica Milenio de MIDEPLAN. 134 p.

Lara A, R Urrutia, C Little, A Martínez. 2010. Servicios ecosistémicos y ley del bosque nativo: No basta con definirlos. Bosque Nativo 47(1): 3-9.

Little C, A Lara, J McPhee, R Urrutia. 2009. Revealing the impact of forest exotic plantations on water yield in large scale watersheds in South-Central Chile. Journal of Hydrology 374: 162-170.

Little C, A Lara. 2010. Restauración ecológica para aumentar la provisión de agua como un servicio ecosistémico en cuencas forestales del centro-sur de Chile. Bosque 31(3): 175178.

Mancilla G, C Valdovinos, M Azocar, P Jorquera, R Figueroa. 2009. Efecto del reemplazo de la vegetación nativa de ribera sobre la comunidad de macroinvertebrados bentónicos en arroyos de climas templados, Chile central. Hidrobiológica 19(3): 193-203.

Martínez E. 1996. La restauración ecológica. Ciencias 43(1): 5661.

Meli P. 2003. Restauración ecológica de bosques tropicales. Veinte años de investigación académica. Interciencia 28(10): 581-589.

Mintegui J, J Robredo. 1994. Caracterización de las cuencas hidrográficas, objeto de restauración hidrológico-forestal, mediante modelos hidrológicos. Ingeniería del Agua 1(2): 69-82.

Naiman R, H Décamps. 1997. The ecology of interfaces: Riparian zones. Annual Review of Ecology and Systematics 28(1): 621-658.

Naiman R, H Décamps, M Pollock. 1993. The role of riparian corridors in maintaining regional biodiversity. Ecological Applications 3(2): 209-212.

Ochoa C. 2007. Usos conflictivos del agua: Búsqueda de solucio- nes. Dyna 74(152): 51-61.

Palma A, R Figueroa, VH Ruiz. 2009. Evaluación de ribera y hábitat fluvial a través de los índices QBR e IHF. Gayana 73(1): 57-63.

Parker IM, SH Reichard. 1997. Critical Issues in Invasion Biology for Conservation Science. In Fiedler PL, PM Kareiva eds. Conservation Biology for the Coming Decade. Nueva York, EEUU. Editorial Chapman and Hall, p. 283-305.

Pellet P, E Ugarte, E Osorio, F Herrera. 2005. Conservación de la biodiversidad en Chile, ¿Legalmente suficiente? La necesidad de cartografiar la ley antes de decidir. Revista Chilena de Historia Natural 78: 125-14.

Peredo-Parada M, F Martínez-Capel, DI Quevedo, AB Hernández-Mascarell. 2011. Implementation of an eco-hydrological classification in Chilean rivers. Gayana 75(1): 26-38.

Scarsbrook M, J Quinn, J Halliday, R Morse. 2001. Factors controlling litter input dynamics in streams draining pasture, pine, and native forest catchments. New Zealand Journal of Marine and Freshwater Research 35: 751-762.

SER. 2004. The SER International Primer on Ecological Restoration. www.ser.org \& Tucson: Society for Ecological Restoration International. 14 p.

Sirombra M, L Mesa. 2010. Composición florística y distribución de los bosques ribereños subtropicales andinos del Río Lules, Tucumán, Argentina. Revista de Biología Tropical 58(1): 499-510.

Steinfeld H, P Gerber, T Wassenaar, V Castel, M Rosales, C de Haan. 2009. La larga sombra del ganado. Problemas ambientales y opciones. Roma, Italia. Organización de las Naciones Unidas para la Agricultura y la Alimentación (FAO). $25 \mathrm{p}$.

Sweeney B, T Bott, J Jackson, L Kaplan, J Newbold, L Standley, W Hession, R Horwitz. 2004. Riparian deforestation, stream narrowing and loss of stream ecosystem services. Proceedings of the National Academy of Sciences of the United States of America 101(39): 14132-14137.

Weisberg P, S Mortenson, T Dilts. 2013. Gallery Forest or Herbaceous Wetland? The need for multi-target perspective in riparian restoration planning. Restoration Ecology 21(1): 12-16.

Young T. 2000. Restoration ecology and conservation biology. Biological Conservation 92(1): 73-83. 